\title{
DENSITOMETRY-BASED FEM SIMULATIONS OF NOVEL POROUS IMPLANTS AND CORRESPONDING STRESS DISTRIBUTION AT THE PERI-IMPLANT AREA
}

\author{
Luboš ŘEhouneK*, Aleš JíRA \\ Czech Technical University in Prague, Faculty of Civil Engineering, Department of Mechanics, Thákurova 7, \\ 16629 Prague 6, Czech Republic \\ * corresponding author: lubos.rehounek@fsv.cvut.cz
}

\begin{abstract}
The presented work focuses on determining the stress distribution at the peri-implant area around dental implants. A numerical analysis simulating the conditions of chewing food has been performed on a FEM model. This model has been created using anonymized real patient CT data and a dental implant model developed at CTU. The CT data served as a 3D geometry and also as a way to construct the global matrix of stiffness of the bone material. Bone density was used as the defining parameter in determining the values of Young's moduli of individual finite elements by the computational program (Mechanical Finder). The implant was introduced as a user-created STL file, which was imported to the computational software and situated inside the geometry of the human mandible. The results show that, as predicted, porous implants achieve higher values of minimum principal stress in the bone as opposed to homogeneous implants (13.4 MPa vs. 7.0 MPa), thus reducing stress shielding.
\end{abstract}

KEYwords: Dental implant, FEM, mechanical finder, porous, stress shielding.

\section{INTRODUCTION}

The dental implants market seems to be one of the most rapidly developing markets in the field of biomaterials [1] and recently, inhomogeneous structures started to emerge as a viable alternative to conventional implants. The goal of this research is to compare different geometrical solutions of a novel porous dental implant with the aid of FEM. The main quantitative measure of this computation is the stress distribution in the surrounding bone and in the body of the implant. The research also aims to evaluate the viability of FEM in regard to simulation of small-scale biomechanical models, such as the human mandible, dentition and dental implants. Our main goal in designing new implants is to reduce stress concentrations, as they are known to be the reason for early implant failure [2], and also introduce a level of porosity into the implant. This approach is possible with the advent of additive manufacturing. Direct metal laser sintering (DMLS) enables for manufacturing of morphologically complex structures, which would otherwise not be possible to create with conventional means of production [3. Porous structures, such as the gyroid structure, demand use of additive manufacturing technologies. The 3D printing of metals and metal alloys serves this purpose well.

The main goal of this study was to try to evaluate the feasibility of the FEM software Mechanical Finder for analysing the human bone system, specifically human dentition. The authors were convinced that based on Wolff's Law [4] developed by the German anatomist and surgeon Julius Wolff, bones which are not subjected to mechanical loading tend to decrease in density and quality. The idea of porous implants is based on this fundamental law so as to provide greater loads to the bone and prevent the effect of stress-shielding. Porous implants, which have a much lower value of global Young's modulus E, present a viable alternative for increasing the stresses in bone at the peri-implant area. As the homogeneous, stiffer implants have much higher E, authors expect the stresses transfered to the bone to be much lower.

\subsection{Porous Implants}

Porosity is welcome as it increases the surface area of the implant, thus creating a better environment for osseointegration, and also enables osteons to grow inside the body of the implant. Bone ingrowth then has the potential to create a better, interconnecting interface of bone and implant material, thus increasing the stability of the implant inside human bone. The structures and whole implants, such as the ones illustrated in Fig. 2 and Fig. 3, are usually designed in a CAD software and subsequently 3D-printed using a metal powder.

The authors of this study already know the possibility of bone ingrowth from their own in-vivo experiments [5] on laboratory pigs, where the osseointegration and BIC (Bone-Implant Contact) were investigated. The magnitude of the pores (or trabeculae) is, therefore, a subject of discussion. Authors list some general clues [6] and their own recommendations for mean pore size, but opinions vary and authors suggest that more in-vivo experiments are needed to ascertain the optimal range of pore size. To give the reader 

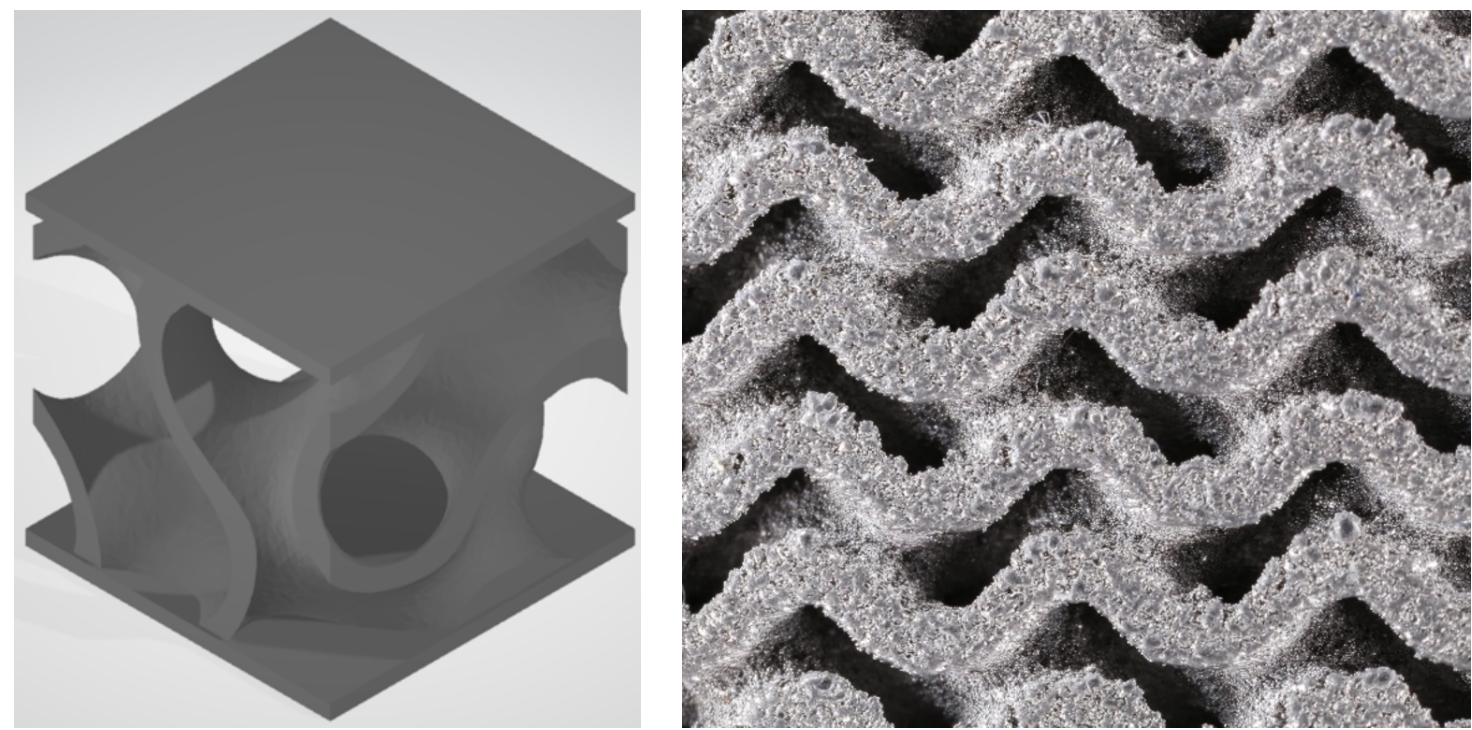

Figure 1. A model of a sample unit cell of the gyroid structure with end blocks (left) and a microscope image of a real, 3D-printed gyroid structure specimen (right) dedicated to macromechanical tests. As the cell is repetitive, the model can be made by multiplication of the unit cell. Material of the print is Ti-6Al-4V.

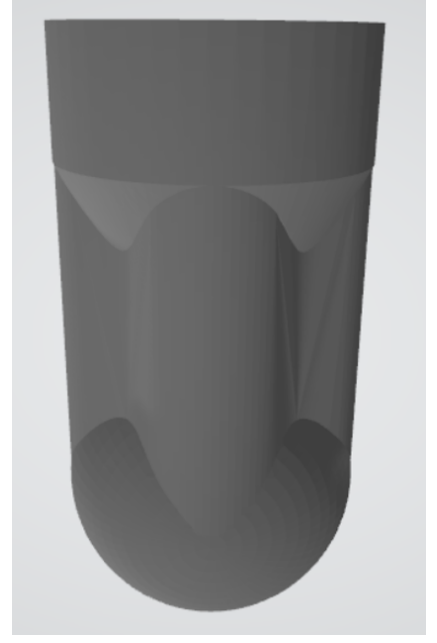

Figure 2. The first, homogeneous geometrical solution of the dental implant used for numerical simulations. Implant was previously patented in the Czech Republic [7. Image shows the intraosseous part.

a good estimate, the values usually fall in the range of 300-900 $\mu \mathrm{m}$.

Authors would also like to address benefits which are special to the gyroid structure. The gyroid structure (Fig. 1) is a special 3D triply periodic structure which is governed by the following equation:

$$
\sin x \cos y+\sin y \cos z+\sin z \cos x=0
$$

Authors want to denote that in their experiments, the gyroid structure seems to be much less prone to crack formation during the process of $3 \mathrm{D}$ printing, as opposed to trabecular structures. One of the main shortcomings of trabecular structures is their

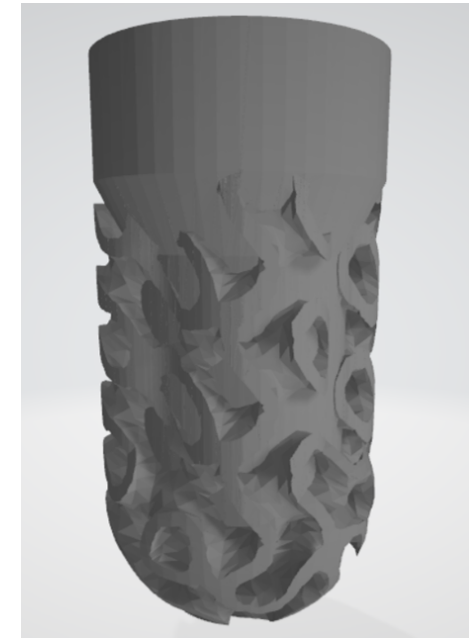

Figure 3 . The second, porous geometrical solution of the dental implant used for numerical simulations. Implant was previously patented in the Czech Republic 7. Image shows the intraosseous part.

beam-like character that contributes to an uneven heat dissipation process. This process has proven itself to be somewhat limiting in the design of trabecular structures. With the gyroid structure, authors have yet to experience a problem of this nature during the production process. Thanks to its wall-like character, the gyroid is also less prone to local damage, crack propagation, formation of debris and manufacturing errors. Especially, debris formation needs to be ruled out in case of dental implants as eroded particles must not enter the body of the patient. Considering osseointegration, one of its benefits is also the absence of sharp edges and corners, which inhibit the circulation of body fluids and the overall healing process. 


\section{Materials AND Methods}

To examine the stress distributions at the peri-implant area, two simulations were performed in the Mechanical Finder software with the aid of a densitometry model of human mandible. The first simulation was done with a homogeneous implant (Fig. 2) and the second with a porous implant with ribs made of a gyroid structure and a homogeneous stem (Fig. 3). The 3D-model of the mandible was created from a CT scan. Material model of the bone is fully inhomogeneous - densitometry assigns values of Young's moduli to every element in the FEM mesh. The software is able to simulate patient-specific conditions this way. A porous dental implant with a system of four stabilizing ribs [7] was situated inside a real CT model of human mandible, constrained and its upper structure loaded with a masticatory force of $150 \mathrm{~N}$.

\subsection{Creating the 3D model}

The creation of the $3 \mathrm{D}$ model begins by taking a calibrated CT scan of the area the user wants to analyze. The model is usually calibrated using the phantom instrument with calibrating rods. After taking the sliced CT data, the user creates a ROI - Range of Interest - they want to specifically include in the model. The process of creating a specific ROI is shown in Fig. 4 This process includes several tools like transparent imaging method, editing multiple slices at once, interpolating between slices and automated editing of ROI.

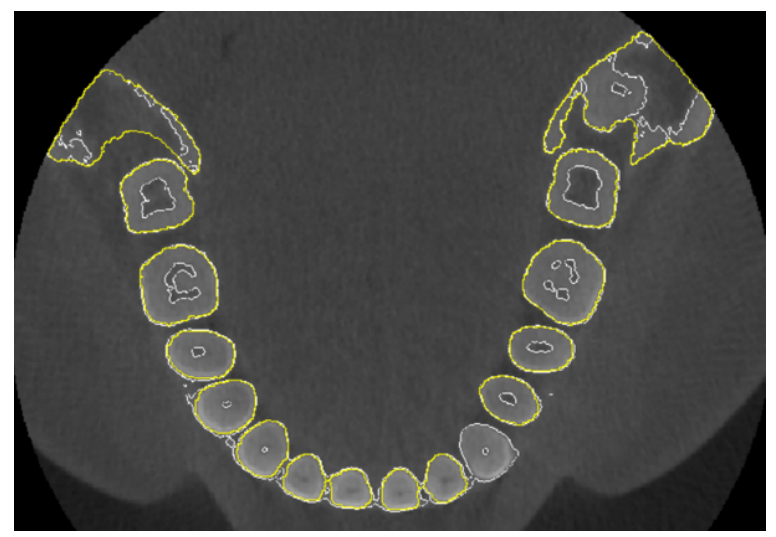

FiguRE 4. User-specified ROI from a patient-specific CT scan. Gray lines indicate the regions recognized by the software from a pre-set threshold value. Yellow lines indicate user-specified ROI (a canine was extracted as a dental implant was placed in its stead.

When the process of ROI extraction is finished, the user can generate a complete $3 \mathrm{D}$ model of the ROI and procede with additional operations, such as subtracting bone parts, cutting the model and adding individual bone parts into separate categories for the analysis. The wireframe model is shown in Fig. 5

The implants can be introduced either by importing an .STL file extension or the .IMP file extension. The

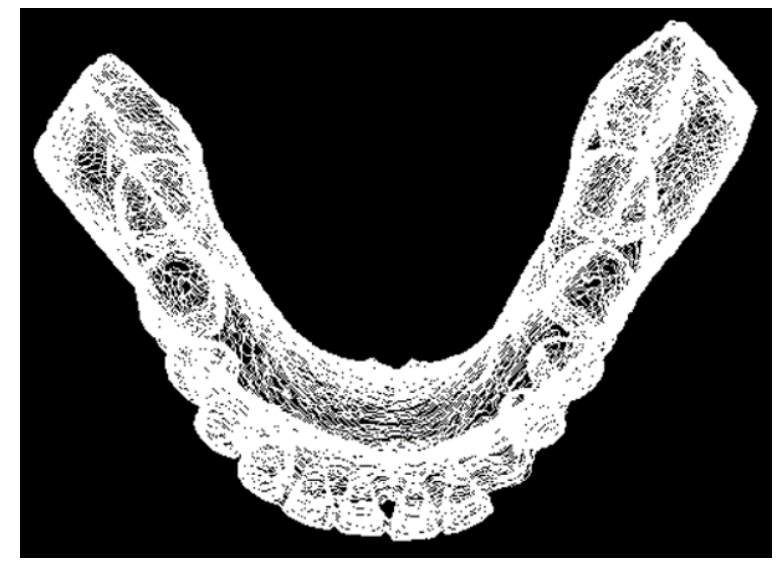

Figure 5. The wireframe model of the human mandible for the creation of the FEM mesh. The left canine was extracted in the step of ROI extraction as dental implants will be introduced.

implant is then placed within the desired position by translation and rotation and/or by using the snap tool or the bone axis tool. In our case, two separate models were created, one for the homogeneous and one for the porous implant variant.

\subsection{LoAd AND Boundary Conditions}

In Mechanical Finder, the user can specify usual boundary conditions by applying restrictions on an individual node and/or neighboring nodes. The force was specified as a total force with a specific vector bound to the local system of coordinates (Fig. 6). The total load on the upper lid of the implant was set to a value of $150 \mathrm{~N}$. This value of force falls within the common range of masticatory forces and does not need to be specific, since the goal of the simulation is to compare the behavior of 2 different implants and the value of force only serves as a constant.

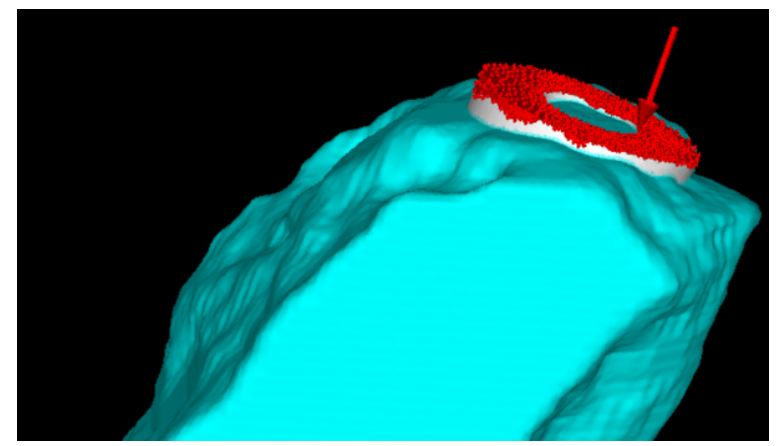

FiguRE 6. Loads and boundary conditions are implemented by selecting individual and neighboring nodes and providing a direction in the form of a vector. 


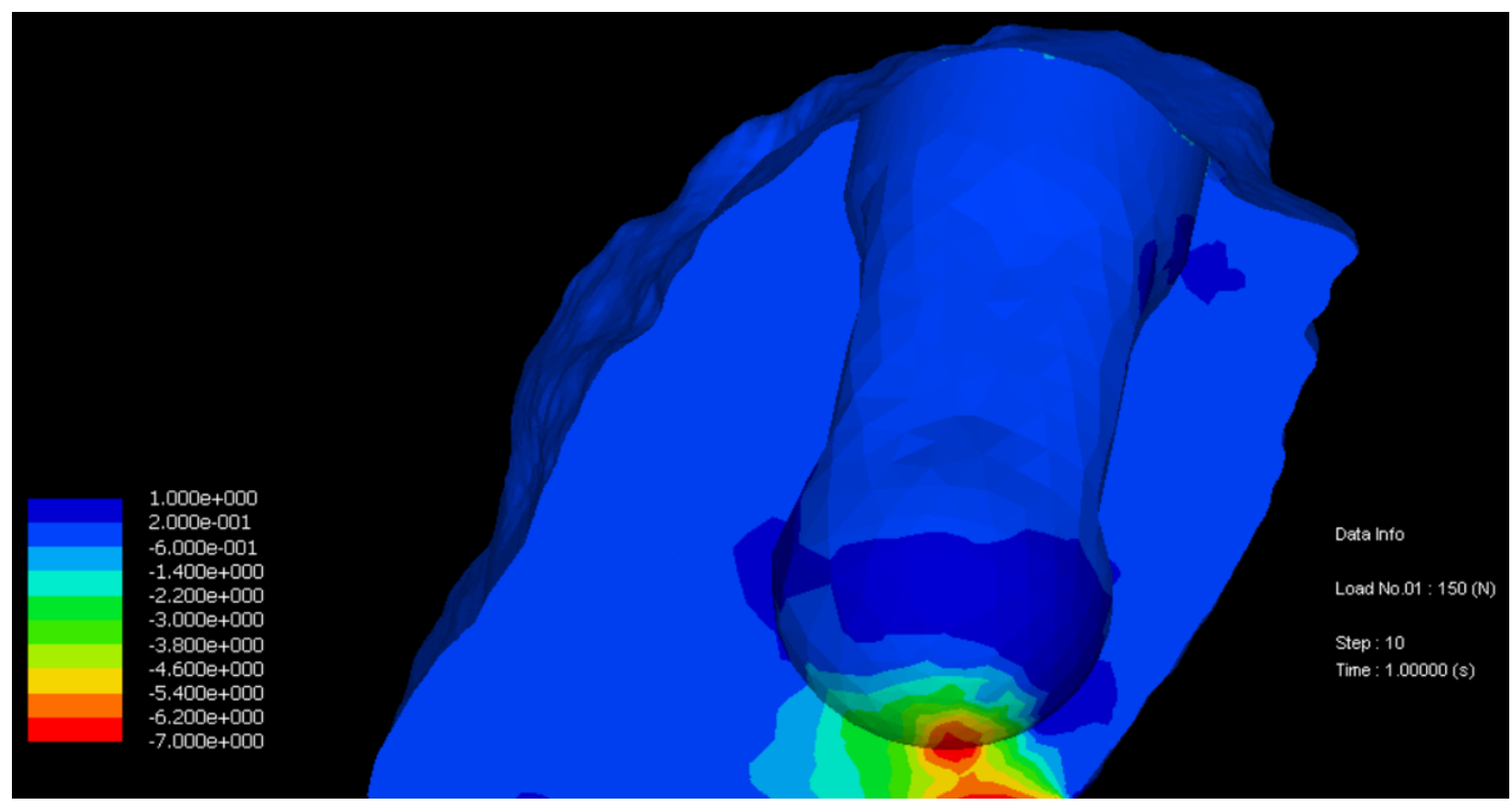

Figure 7. The minimum principal stress of the bone material at the peri-implant area in MPa for the homogeneous variant of the implant. The maximum value of compressive stress at this section is $7.0 \mathrm{MPa}$ for an axial load of $150 \mathrm{~N}$. The value is smaller as compared to Fig. 8 because the implant is much stiffer and transfers much less load into the surrounding bone.

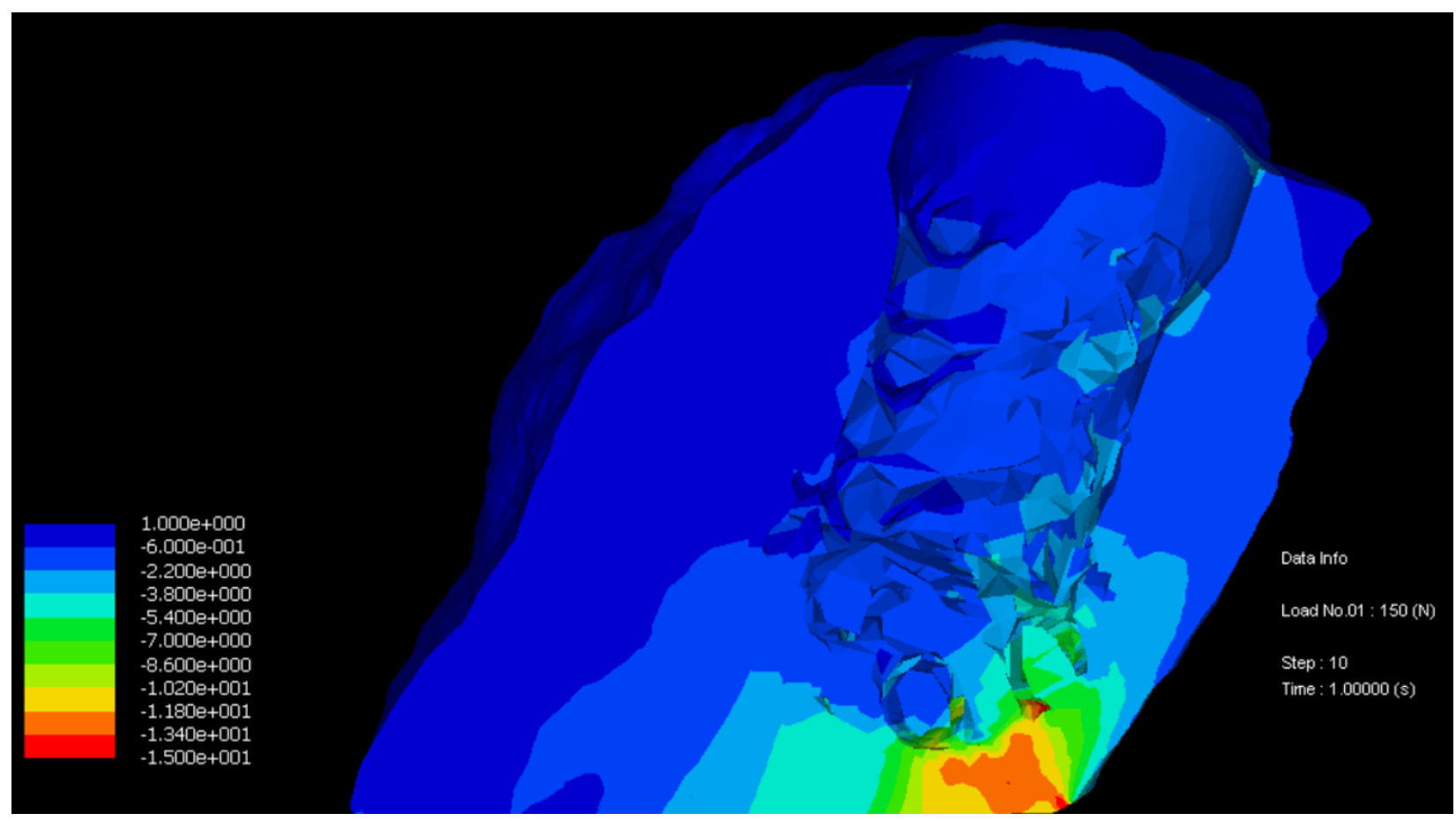

FiguRE 8. The minimum principal stress of the bone material at the peri-implant area in MPa for the porous variant of the implant. The maximum value of compressive stress at this section is $13.4 \mathrm{MPa}$ for an axial load of $150 \mathrm{~N}$. The value of stress is higher as compared to Fig. 7 because lower stifness of the porous implant provides the peri-implant area with more load, thus increasing the stress. 


\subsection{Numerical Simulation}

Two simulations were performed on the same human mandible model - one for the homogeneous (Fig. 2) and one for the porous (Fig. 3) implant. The simulations were performed with the same boundary conditions and exactly the same load. The goal of the simulations was to verify the hypothesis that a porous implant would transfer more load into the bone at the peri-implant area, thus reducing the effect of stressshielding and subsequent bone loss.

The material model of the bone which was used for this simulation was the Keyak model [8]. The material used for the imported .STL implant model file was Ti-6Al-4V, which is a common implant material in the industry. The comparison was carried out by comparing the values of minimum principal stress of the bone at the peri-implant area. Note that greater values of stress are beneficial in this case, as bone has high compressive strength, but often lacks mechanical stimulation when an implant is introduced, leading to bone loss and aseptic loss of stability and failure of the implant [2].

\section{Results}

The results of the numerical analyses suggest that the second implant variant with a porous outer layer and ribs provides greater values of stress inside the bone under the same load conditions (13.4 MPa peak minimum principal stress for the porous implant and 7.0 MPa for the homogeneous implant). This finding supports the hypothesis laid out by the authors. The distribution of minimum principal stress in the bone of human mandible are shown in Fig. 7 and Fig. 8 for the homogeneous and porous geometrical variant, respectively.

The difference in the values of minimum principal stress arises from different values of global moduli of both implants. It is very important to distinguish between the modulus on the microscale (the modulus of the print material Ti-6Al-4V) and the modulus of a homogeneous structure and a porous structure, such as the one shown in Fig. 11 The modulus of the print material is $\mathrm{E}_{\mathrm{Ti}-6 \mathrm{Al}-4 \mathrm{~V}}=120 \mathrm{GPa}$ in both cases, but the global modulus of the gyroid structure itself is expected to be approximately 1 order of magnitude lower (authors have not yet done mechanical tests on this exact presented structure but have experience testing similar structures [9]).

\section{Conclusions}

Numerical analyses show that by incorporating some degree of porosity into an implant, we can reduce the effect of stress shielding on the human bone. As presupposed, the homogeneous implant transfers less load into the bone when compared to the same implant with a porous outer layer. This fact can be attributed to lower overal stiffness at the peri-implant area. Greater values of compressive stress (13.4 MPa for the porous implant vs. 7.0 MPa for the homogeneous one) are beneficial as they provide the bone with more load to maintain its density and prevent bone loss, as described by the Wolff's Law [4]. Another benefit of the structure is its ability to let osteons grow inside the body of the implant and provide an interlocking mechanism which can potentially better the primary stability of the implant. With the advancement of additive manufacturing technologies, such complex structures are becoming easier to manufacture and more financially accessible to the general population.

When evaluating porous structures, authors also see the potential of the gyroid structure as compared to other porous structures, such as the trabecular structure - ease of manufacture during the 3D printing process and the lack of sharp edges and discontinuities between individual beams are beneficial to the overall quality of the final product.

\section{ACKNOWLEDGEMENTS}

The financial support by the Faculty of Civil Engineering, Czech Technical University in Prague (SGS project No. SGS17/168/OHK1/3T/11) is gratefully acknowledged. The authors also gratefully acknowledge the support granted by the Czech Ministry of Industry and Trade (project OP PIK No. CZ.01.1.02/0.0/0.0/17_102/0011518).

\section{REFERENCES}

[1] C. M. Abraham. Suppl 1: A brief historical perspective on dental implants, their surface coatings and treatments. The open dentistry journal 8:50, 2014.

[2] M. Ridzwan, S. Shuib, A. Hassan, et al. Problem of stress shielding and improvement to the hip implant designs: a review. J Med Sci 7(3):460-467, 2007.

[3] A. El-Hajje, E. C. Kolos, J. K. Wang, et al. Physical and mechanical characterisation of 3D-printed porous titanium for biomedical applications. Journal of Materials Science: Materials in Medicine 25(11):24712480, 2014. DOI:10.1007/s10856-014-5277-2.

[4] S. Cowin, D. Hegedus. Bone remodeling i: theory of adaptive elasticity. Journal of Elasticity 6(3):313-326, 1976.

[5] L. Rehounek, A. Jíra. Numerical and mechanical analyses of a 3d-printed titanium trabecular dental implant. Acta Polytechnica 57(3):218-228, 2017.

[6] S. A. Yavari, J. van der Stok, Y. C. Chai, et al. Bone regeneration performance of surface-treated porous titanium. Biomaterials 35(24):6172-6181, 2014.

[7] A. Jíra, F. Denk, F. Denk. A dental implant shaft, 2017. Czech patent n. 306457.

[8] J. H. Keyak, S. A. Rossi, K. A. Jones, H. B. Skinner. Prediction of femoral fracture load using automated finite element modeling. Journal of biomechanics 31(2):125-133, 1997.

[9] L. Řehounek, P. Hájková, P. Vakrčka, A. Jíra. Geometry and mechanical properties of a $3 \mathrm{~d}$-printed titanium microstructure. Acta Polytechnica CTU Proceedings 15:104-108, 2018. 